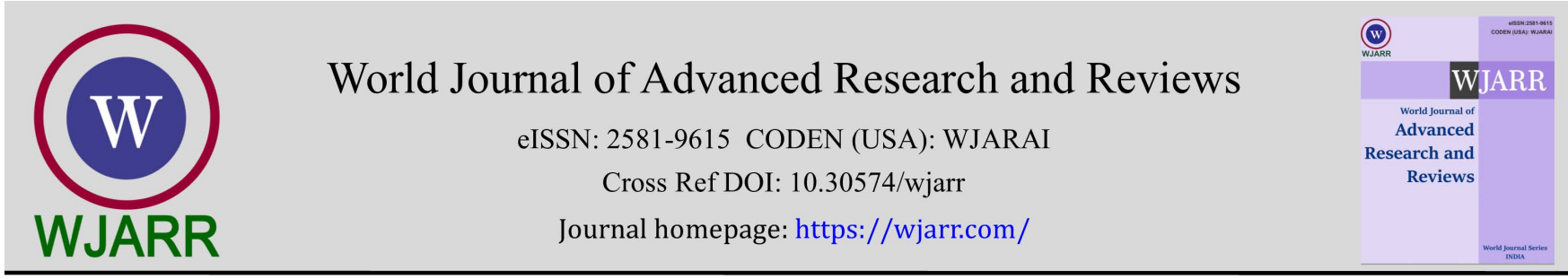

(REVIEW ARTICLE)

Check for updates

\title{
Anatomical variation of the median nerve and its thenar branch: Clinical and surgical application in the treatment of carpal tunnel syndrome
}

\author{
Charaf eddine Elkassimi *, Mustapha Fadili, Sami Rouadi and Abdelhak Garch \\ Anatomy laboratory, Faculty of Medicine and Pharmacy of Casablanca, Hassan II University, Morocco.
}

World Journal of Advanced Research and Reviews, 2021, 09(03), 326-329

Publication history: Received on 13 February 2021; revised on 18 March 2021; accepted on 21 March 2021

Article DOI: https://doi.org/10.30574/wjarr.2021.9.3.0102

\begin{abstract}
Carpal tunnel syndrome is the most common root canal pathology. The surgical treatment corresponds to a release of the median nerve by incision of the anterior annular ligament of the carpus by open surgical treatment or endoscopy.

Knowledge of the normal anatomy and anatomical variations of the median nerve at the wrist is fundamental to avoiding complications in median nerve release in the treatment of carpal tunnel syndrome.

Through this work we will show the interest of knowledge of the anatomy of the median nerve as well as its anatomical variations in order to derive the main clinical applications and to avoid the risks associated with open or endoscopic surgery in the treatment of carpal tunnel syndrome.
\end{abstract}

Keywords: Median nerve; Anatomical variation; Carpal tunnel

\section{Introduction}

The compression of the median nerve at the wrist or Carpal tunnel syndrome is the most frequent enclaving neuropathy, described in 1913 by Marie and Foix as a cause of the syndrome of "nocturnal brachialgia paresthesia"[1].

The anatomical course of the thenar motor branch of the median nerve is highly variable. Injury to this branch during carpal tunnel syndrome surgery can lead to a functional deficit in the thumb.

Minimally invasive surgical techniques and endoscopic procedures in the treatment of carpal tunnel syndrome require a thorough and perfect knowledge of the anatomy and variations of the anatomical structures of the carpal tunnel.

The goal of our work through a review of the literature is to show the value of knowledge of the anatomy of the median nerve as well as its anatomical variations in order to draw the main clinical applications and avoid the risks associated with open or endoscopic surgery in the treatment of carpal tunnel syndrome.

\section{Carpal tunnel surgical anatomy}

The carpal tunnel is an osteofibrous, inextensible tunnel which corresponds to the proximal part of the palmar region of the hand.

\footnotetext{
${ }^{*}$ Corresponding author: Charaf Eddine Elkassimi

Anatomy laboratory, Faculty of Medicine and Pharmacy of Casablanca, Hassan II University, Morocco. 
It is located between the lower transverse crease of the wrist and a horizontal line approximately $3.5 \mathrm{~cm}$ lower. Its surface is $5 \mathrm{~cm}^{2}$ in its proximal part and $3 \mathrm{~cm}^{2}$ in its distal part.

This canal having the shape of a diabolo, is delimited by the eight carpal bones, and closed on the ventral surface by the flexor retinaculum (formerly called the anterior annular ligament of the carpal which is inserted laterally on the scaphoid and trapezius, and medially on the pisiform and hamatum, transforming this space into an inextensible canal: the carpal tunnel.

In this space, with fixed dimensions, the median nerve runs along with nine tendons; four flexor digitorum superficialis tendons, four flexor digitorum deep and flexor digitorum longus tendon, these are sometimes accompanied by a persistent median artery.

The median nerve arrives at the level of the wrist, passing between the tendon of the flexor carpi radialis and that of the palmar longus. It then engages the carpal tunnel by sliding against the deep surface of the anterior annular carpal ligament.

During the wrist extension movement, the median nerve is placed between the flexor retinaculum and the flexor digitorum superficial tendons. Wrist flexion produces an anterior displacement of the flexor tendons towards the flexor retinaculum, which is why wrist flexion rather than extension is considered a predisposing mechanism for carpal tunnel syndrome, although carpal tunnel pressure increases with the two movements $[2,3]$.

The median nerve normally terminates at the lower edge of the flexor retinaculum in two lateral and medial trunk which give rise to 6 branches, including the motor thenar branch. This thenar branch is recurrent and innervates the abductor pollicis brevis muscles of the thumb, the opposing thumb and the superficial head of the flexor digitorum brevis muscle [4].

Since the origin of the recurrent motor branch in the carpal tunnel is along the thenar flexion crease, the surgical incision to expose the carpal tunnel or to incise the anterior annular ligament of the carpal tunnel should be ulnar at this crease and the annular ligament underlying.

There is usually a sensory communicating branch between the ulnar and median nerve in the hand involved in the innervation of the 3rd and 4th finger. This branch, called the anastomotic branch of Berrettini, usually crosses the superficial palmar vascular arch, but in some cases it may sit further upstream, just at the level of the distal edge of the anterior annular carpal ligament. This position makes her vulnerable to injury during carpal tunnel release [5].

The deep motor branch of the ulnar nerve passes next to the ulnar edge of the hook of the hamatum. If the ulnar side of the flexor retinaculum is incised during the treatment of carpal tunnel syndrome trying to avoid the median nerve, the motor branch of the ulnar nerve may be injured.

\section{Discussion}

Variable results have been reported on the prevalence of anatomical abnormalities of the median nerve. These anatomical variations are well described because of their diagnostic and surgical importance $[6,7,8]$.

Lanz classified the anatomical variations of the thenar branch of the median nerve into four groups:

Group 0: extra ligament thenar branch (standard anatomy)

Group I: Variations in the path of the thenar branch, this group is subdivided into four subgroups.

Group Ia (sub ligament): the motor branch of the median nerve begins under the anterior annular ligament of the carpal and then folds around its distal edge.

Group I b (Trans ligamentary): the motor branch born on the radial side of the median nerve then passes through the annular ligament.

Group I c: the motor branch comes from the ulnar side of the median nerve. 
Group I d (supraligamentary): the motor branch folds around the distal edge of the ligament

The trans ligament variety is of great clinical importance due to the possibility of compression within the retinacular fibers which will result in carpal tunnel syndrome.

Group II: Accessory branch of the median nerve at the distal part of the carpal tunnel

Group III: High level of division of the median nerve

Group IV: Accessory branch of the median nerve at the proximal part of the carpal tunnel [9].

According to the different series published in the literature, the most common anatomical variety of the thenar motor branch of the median nerve was extra ligament with an estimated average prevalence of $73.8 \%$.

The results of an intraoperative study conducted by Green and Morgan, found in 74.5\% of carpal tunnel syndromes operated on, a sub ligamentous path. Their results differ considerably from the rest of the published series and this contradiction could be due to the intraoperative nature of the study, in which the visual field during surgery could hamper the ability to accurately identify the path of the thenarian motor branch of the median nerve $[10,11]$.

The clinical or electrophysiological signs of the presence of anatomical variations of the median nerve in the carpal tunnel in a patient are rare or even nonexistent, so on clinical examination it will be difficult to determine with precision the anatomical variety of the thenar branch of the median nerve [12.13].

According to the literature the prevalence of the trans ligament variety of the thenar motor branch of the median nerve is $12.1 \%$. This variety has the highest risk of accidental injury during carpal tunnel syndrome surgery, which is why most surgeons recommend the use of an ulnar surgical approach, with dissection careful plan by plan of the carpal tunnel to avoid neurovascular damage [14].

Subligamentous path of the thenar motor branch of the median nerve also presents an increased risk of injury during surgical procedures, and care should be taken to identify the presence of this variation [15].

In addition, the presence of hypertrophic muscles interposed on the anterior annular carpal ligament should serve as a warning sign to the surgeon of potential variants of the thenar motor branch of the median nerve [6].

Lanz reported that the presence of a bifid median nerve was often associated with the presence of a persistent median artery circulating between the two parts of the bifid nerve [9].

The median artery during embryonic life is the main source of blood supply to the hand, but it regresses throughout foetal development. A persistent median artery, when present, supplies blood to the superficial palmar arch or radial fingers in addition to the median nerve [16]. Damage to this artery during carpal tunnel syndrome surgery can significantly affect blood flow to the hand [17].

Since a bifid median nerve and a persistent median artery are common anatomical variations and are often associated with other variations of the median nerve, ultrasound screening of patients before undergoing surgery for carpal tunnel syndrome can help identify those that present an increased risk of iatrogenic nerve damage and allow for better surgical planning [18].

\section{Conclusion}

Anatomical variations of the median nerve are frequent and present a risk of iatrogenic surgical lesions if they are not recognized.

Good management of carpal tunnel syndrome requires a thorough knowledge of the anatomy of the median nerve and its possible anatomical variations.

The preoperative use of ultrasound can help identify patients with a bifid median nerve or a persistent median artery, who are more likely to have other anatomical variations of the median nerve. 


\section{Compliance with ethical standards}

\section{Acknowledgments}

The authors would like to thank anonymous reviewers for constructive comments on the manuscript.

\section{Disclosure of conflict of interest}

The authors declare that there is no conflict of interest.

\section{References}

[1] Samson P. Le syndrome du canal carpien. EM Consulte. 2004; 23(S1): 165-177.

[2] Gelberman RH, Szabo RM, Mortensen WW. Carpal tunnel pressures and wrist position in patients with colles' fractures. J Trauma. 1984; 24(8): 747-749.

[3] Krishnan P, Mishra R, Jena M, Das A. Transligamentous thenar branch of the median nerve: the million dollar nerve. Neurol India. 2013; 61(3): 311-2.

[4] Schmidt HM, Normal anatomy and variations of the median nerve in the carpal tunnel . Verlag Berlin Heidelberg journal . 2007; 13-20.

[5] May JW Jr, Rosen H. Division of the sensory ramus communicans between the ulnar and median nerves: A complication following carpal tunnel release. A case report. J Bone Joint Surg Am. 1981; 63(5): 836-838.

[6] Alizadeh K, Lahiji F, Phalsaphy M. Safety of carpal tunnel release with a short incision. A cadaver study. Acta Orthop Belg. 2006; 72(4): 415-419.

[7] Engineer NJ, Hazani R, Mowlavi A, Neumeister MW, Lee WPA,Wilhelmi BJ. Variations in the anatomy of the third common digital nerve and landmarks to avoid injury to the third common digital nerve with carpal tunnel release. Eplasty. 2008; 8e: 51.

[8] Beris AE, Lykissas MG, Kontogeorgakos VA, Vekris MD, Korompilias AV . Anatomic variations of the median nerve in the carpal tunnel release. Clin Anat. 2008; 21: 514-518.

[9] Lanz U. Anatomical variations of the median nerve in the carpal tunnel. J Hand Surg Am. 1977; 2(1): 44-53.

[10] Green DP, Morgan JP. Correlation between muscle morphology of the transverse carpal ligament and branching pattern of the motor branch of median nerve. J Hand Surg Am. 2008; 33(9): 1505-11.

[11] Falconer D, Spinner M. Anatomic variations in the motor and sensory supply of the thumb. Clin Orthop Relat Res. 1985; (195): 83-96.

[12] Demircay E, Kabatas S, Cansever T, Yilmaz C. An anatomical variation of the third common digital nerve and recurrent motor branch of the median nerve. Neurol India. 2009; 57(3): 337-9.

[13] Rodriguez R, Strauch RJ. The middle finger flexion test to locate the thenar motor branch of the median nerve. J Hand Surg Am 2013; 38(8): 1547-50.

[14] Elsaftawy A, Gworys B, Jabłecki J, Szajerka T. "Dangerous" anatomic varieties of recurrent motor branch of median nerve. Pol Przegl Chir. 2013; 85(8): 419-23.

[15] Barbe M, Bradfield J, Donathan M, Elmaleh J. Coexistance of multiple anomalies in the carpal tunnel. Clin Anat. 2005; 18: 251-259.

[16] Al-Qattan MM, Al-Zahrani K, Al-Omawi M. The bifid median nerve re-visited. J Hand Surg Eur. 2009; 34(2): 212214.

[17] Agarwal P, Gupta S, Yadav P, Sharma D. Cadaveric study of anatomical variations of the median nerve and persistent median artery at wrist. Indian J Plast Surg. 2014; 47(1): 95-101.

[18] Bagatur AE, Yalcinkaya M, Atca AO. Bifid median nerve causing carpal tunnel syndrome: MRI and surgical correlation. Orthopedics. 2013; 36(4): e 451-6. 